Nephron 1994;68:524-525

\title{
Subject Index Vol. 68, 1994
}

Abdominal surgery 334

Acidosis 234

Acquired immunodeficiency syndrome

262 Activated partial thromboplastin time 489 Active vitamin D 221 Acute renal failure 63, 128, $327,433,454$

- $\quad$ - transplant rejection 378

Acyclovir 262

Adhesion molecules 192

Adriamycin 347, 353

Albumin 353

Aldosterone 112

Allopurinol 468

Aluminium 197

Amino acids 338

Angiomyolipoma 256

Ankylosing spondylitis 314

Antineutrophil cytoplasmic antibody 380

Antiphospholipid syndrome 128

o1/8-Antiplasmin 489

Antithrombin III 489

Aortic stenosis 159

Apoptosis 104

Atrial natriuretic factor 301,427

- $\quad$ - peptide 112

Autoimmune disease 314

Autonomic neuropathy 301

Bacteremia 314 Bacterial killing 338 Bacteriuria 314 Bicarbonate selectivity 234 Blood pressure 419, 427, 462

- $\quad$ viscosity 184

Cadmium 104

Calcification, extra-articular 159

Calcium 112,197,419,481

CD4 subsets 20

Cell membrane potential 234

- $\quad$ transfer 360

Chemical parathyroidectomy 217

Chemotactic factor 308

Cholesterol 57

Chronic dialysis patients, survival rates 80 
fatigue 314

renal disease 48

- failure 63, 197,202

- -, progression 462 Ciclosporin 57, 375, 497 Citrate 197 Coagulation factors 489

Comorbid condition chronic dialysis

patients 80 Continuous ambulatory peritoneal dialysis

$125,334,437$ Cortical infarction 378

- $\quad$ necrosis 378

Corticosteroids 184

Cyclophosphamide 380

Dialysate temperature 427

ultrafíltration 229 Dialysis 500

membrane permeability 202 Diltiazem 454 Dimethylmethylene blue 449

Echocardiography 301

Electrolytes 112

ß-Endorphin 366

End-stage renal disease 329

Enzyme-linked immunosorbent assay 25

Eosinophilia 259

Epidermal growth factor receptor 97

Erythrocytes 71,419

Erythropoietin 419

E selectin 294

Experimental diabetes 245

Extracellular matrix 353

Eye disease 52

Fibrinogen 489 Foam cells 473

Gentamicin 468

Glanzmann's thrombasthenia 442

Glomerular endothelium 308

epithelial cell line (SGE1) 97

filtration rate 454

mesangial cells 366

mesangium 308 Glomerulonephritis 32,314,497 Glomerulosclerosis 473,481

-, focal 347 -, focal-segmental 497 Glycoprotein lb 442 Glycosaminoglycans 449 Glycosylation 207 Goodpasture's syndrome 52 Granulocytes 192 Growth factors 97

Hantavirus 48 Hematuria 180

Hemodialysis 38, 71, 77, 133, 192, 202,

301,427,442 Hemolytic-uremic syndrome 63, 433 Hemorheology 184,419 Hemorrhagic fever, renal syndrome 48 Hemostasis 184 Heparin 133

Hepatitis $\mathrm{C}$ virus antibodies 38 Hepatocyte growth factor 118 Herpes varicella-zoster virus infection 262 High-protein meal 112 Histones 25 HLA antigens 433 Hybridization, in situ 10

Hyperlipidemia 239, 347, 473 Hypertension 32,314 -, renal disease 48 Hyponatremia 252, 262 -, hypertensive syndrome 252 Hypotension 301

IgA antihistone 25

levels, serum 20 
nephropathy 20, 25, 294, 308 IgM nephropathy 10 Immunity, cell-mediated 360 Inappropriate antidiuretic hormone secretion, syndrome 262

Inflammation 229 Inflammatory bowel disease 314 Insulin 329

secretion 41 Insulin-like growth factor I 481 Intercalated cells 118 Intercellular adhesion

molecule-1 294 Interleukin(s) 10,437

receptor antagonist (IL-IRa) 229

Jugular venous catheters, guide wire 77

K-Cl cotransport 71 Kidney transplant 497 Kimura's disease 259

Lactate 338

Laser photocoagulation 52

Leukocytoclastic angiitis 380

Lipids 462

Low molecular weight heparin 202

Low-protein meal 112

Macrophages 473 Membranous nephropathy 87

524

Mesangial cells 10,481

- $\quad$ interposition 308

Microalbuminuria 169

Minimal-change disease 259

Mizoribine 380

Morphine 366

Necrotizing glomerulonephritis 380 Nephritis, nephrotoxic serum 360 Nephrosis, experimental 353 Nephrotic proteinuria 32

- $\quad$ syndrome $184,239,259,314,489$

Nitro blue tetrazolium reaction 207

Non-A, non-B hepatitis 38

Non-insulin-dependent diabetes mellitus

207 Norepinephrine 301

Omentectomy 327

Opioid receptors 366

Oral calcitriol pulse therapy 221

Osteoarthritis 314

Oxalocalcic urolithiasis 449

Oxidative metabolism 338

Parathyroid gland 221

hormone 419

hyperplasia 221

Percutaneous fine-needle ethanol injection 217

transluminal angioplasty 252 Pericardial effusion 125 Pericardiocentesis 125 Peritoneal dialysate 338

macrophage 338,437 Peritoneopericardial communication 125

Phagocytosis 338 Plasma endothelin-1 372

- $\quad$ renin activity 112

Plasminogen activator inhibitor 1 concentration, fibrinolytic activity 239 Platelet aggregation 133 Platelet-derived growth factor 308, 481 Prednisolone 380 Primary glomerulonephritis 25 
Principal cells 118 Protein loading 169 Proteinuria 353, 462 Prothrombin time 489 Proximal tubules 104 Puromycin aminonucleoside 489 Pyelonephritis 314

Radioisotope imaging 265

$86 \mathrm{Rb}$ uptake 71

Renal artery occlusion 265

blood flow autoregulation 245

failure $159,239,256$

-, progression 32

function 212,454

hemodynamics 245

mass 500

microangiopathy 128

oncocytoma 375

transplant 378

transplantation 57, 375 Renovascular hypertension 252,372 Retinoicacid 97

Retinoids 97 Reviparine 202 Rheumatoid arthritis 314

Salt-induced hypertension 385

Secondary hyperparathyroidism 221

Selective renal venous blood level 372

Simvastatin 57

Skin necrosis 133

Spontaneously hypertensive rat 385,468 ,

481 Streptococcal cell wall 314 Subretinal neovascularization 52 Sugar-induced hypertension 385 Surgical revascularization 265

Terminal complement complex (C56-9) 87 Tertiary hyperparathyroidism 217 Thrombocytopenia

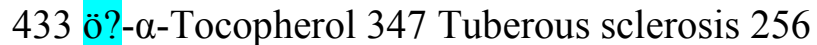

Ultrasonography 221,378,500 Unilateral nephrectomy 462 Uninephrectomized patients, renal function 169 Uremia 41,217,442 Urinary albumin 212

- Bî-microglobulin 212

Urodilation 454

Urokinase 77

Vascular cell adhesion molecule-1 294

Vessels 207

Vitamin D deficiency 41

E 347 Vitronectin 87

receptor 87

Wegener's granulomatosis 380,500

525 